This is a postprint version of the following published document:

Sánchez-Sanz, M. ; Fernández-Galisteo, D. ; Kurdyumov, V. N. Effect of the equivalence ratio, Damköhler number, Lewis number and heat release on the stability of laminar premixed flames in microchannels. Combustion and Flame, 2014, 161 (5), 1282-1293. Available at: http://dx.doi.org/10.1016/j.combustflame.2013.11.015

(C)2013 The Combustion Institute. Published by Elsevier Inc.

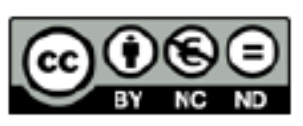

This work is licensed under a Creative Commons Attribution-NonCommercialNoDerivatives 4.0 International License. 


\title{
Effect of the equivalence ratio, Damköhler number, Lewis number and heat release on the stability of laminar premixed flames in microchannels
}

\author{
Mario Sánchez-Sanz ${ }^{\text {a,* }}$, Daniel Fernández-Galisteo ${ }^{\text {b }}$, Vadim N. Kurdyumov ${ }^{\text {b }}$ \\ ${ }^{a}$ Dept. Ingeniería Térmica y de Fluidos, Universidad Carlos III de Madrid, 28911 Leganés, Spain \\ ${ }^{\mathrm{b}}$ Department of Energy, CIEMAT, Avda. Complutense 40, 28040 Madrid, Spain
}

\begin{abstract}
The effect of the equivalence ratio on the stability and dynamics of a premixed flame in a planar microchannel with a step-wise wall temperature profile is numerically investigated using the thermo-diffusive approximation. To characterize the stability behavior of the flame, we construct the stability maps delineating the regions with different flame dynamics in the inlet mass flow rate $m$ vs. the equivalence ratio $\phi$ parametric space. The flame stability is analyzed for fuels with different diffusivity by changing the Lewis numbers in the range $0.3 \leqslant L e_{F} \leqslant 1.4$. On the other hand, the Lewis number of the oxidizer is kept constant and equal to unity $L e_{O}=1$. Our results show that, for very diffusive fuels, the stability of the flame varies significantly with the equivalence ratio, transitioning from stable flames for lean mixtures to highly unstable flames when $\phi>1$. As the fuel Lewis number approaches unity, the stability behavior of the flame for lean and rich mixtures becomes more similar to give, in the equidiffusional case $L e_{F}=1$, a symmetric stability map around the stoichiometric mixture $\phi=1$. In all cases considered, the most stable flames are always found around the stoichiometric mixtures $\phi=1$, when the flame instabilities are completely suppressed for very diffusive fuels $L e_{F}<1$, or are reduced to a narrow range of inflow velocities for fuel Lewis numbers equal or greater than unity.

The ratio between the size of the channel and the flame thickness $d$ turns out to be of great importance in the stability behavior of the flame. Keeping the rest of parameters constant, an increase in $d$ for lean flames makes the flame considerably more unstable, confirming the findings of previous works. Nevertheless, as the stoichiometric ratio approaches $\phi=1$, that trend is reversed to give flames that become more stable as the size of the channel is increased.
\end{abstract}

Keywords: Differential diffusion, Microchannel combustion, Symmetric and Non-symmetric flames Oscillatory, instability, Flame dynamics, Solution multiplicity.

\section{Introduction}

The improvement of the fabrication techniques has encouraged the miniaturization of mechanical and electro-mechanical devices, following the trend previously initiated by other technological areas, such as electronics or biomechanics [1]. The primary objective of using fossil fuels to power these system is to leverage the high energy density of fuels, specifically liquid hydrocarbon fuels, relative to batteries and all other energy storage devices. As such, considering $45 \mathrm{MJ} / \mathrm{kg}$ as an average liquid fuel energy density, a miniaturized device with a power conversion efficiency of around $2-5 \%$ would compete with the energy density of around $2.5 \mathrm{MJ} / \mathrm{kg}$ found on the top Lithium/thionyl chloride primary batteries currently available in the market or the average $0.6 \mathrm{MJ} / \mathrm{kg}$ of an Alkaline battery [1].

\footnotetext{
* Corresponding author.

E-mail address: mssanz@ing.uc3m.es (M. Sánchez-Sanz).
}

According to Dunn-Rankine et al. [2], to be useful as a portable power device, a system needs to achieve power densities between 10 and $1000 \mathrm{~W} / \mathrm{kg}$ and working autonomies between 1 and $10 \mathrm{~h}$. Among the different technologies capable of achieving such figures, we find that heat engines and, specially, rotary engines, are specially well suited for small-power generation due to its high specific power, low cost and minimum number of moving parts [3].

In a small-scale rotary engine, a triangular rotor revolves inside the epitrochoidal housing. The rotor admits a combustible mixture, with a given equivalence ratio, that is mechanically compressed to reach a maximum pressure when the volume of the region between the rotor and the housing is minimum near the top dead center. At that precise instant, the mixture is ignited and the flame propagates along a slender volume of width of the order of the flame thickness and length around 20 times the chamber width $[3,6]$.

The experimental feasibility of these small-scale engines has been already proven by $\mathrm{Fu}$ and coworkers $[4,5]$. They built a rotary 
engine working with Methanol-Nitromethane mixtures with an equivalence ratio $\phi=0.4,138 \mathrm{~mm}^{3}$ of average displacement, power output around $100 \mathrm{~W}$ and thermal efficiency below $0.5 \%$, very far from the minimum needed to make the engine an actual competitor of batteries. Larger efficiencies could have been obtained with a fuel/air ratio closer to stoichiometry, but unresolved combustion-related problems hampered the normal operation of the engine [4].

The reduction of the engine size brings new challenges that needs to be specifically treated to improve the engine performance, such as increasing heat losses, flame instabilities or flame quenching. The resemblance of the geometry of the experiments carried out by Tsuji et al. [6] and Sprague et al. [3] with a narrow, straight channel of great length, has motivated a great number of theoretical studies based in this simple configuration in which the particularities of the combustion in the small scale have been analyzed. Specifically, Ju and Xu [7] demonstrated that, for flames propagating in narrow channels, the increasing of the surface-to-volume ratio is associated with a rise in the convective heat losses to the walls of the channel proportional to the square of the ratio of flame thickness $\delta_{T}$ to the channel width $h$. Furthermore, for a constant mass flow rate, a reduction in the channel size is inevitably associated with smaller residence times, what contributes to diminish further the Damkhôler number of the combustion reaction and makes the flame more prompt to quench and/or develop instabilities that make the system difficult to control [8].

The thermal coupling of methane and propane flames with the walls of a straight micro-channel with a step-wise wall temperature profile revealed a complex flame dynamics $[9,10]$. These works showed that stoichiometric or near-stoichiometric mixtures give stable flames in the high and low incoming velocity regions and unstable flames in the middle velocity region, with flame extinctions and re-ignitions that are repeated periodically in time. A similar instability has been observed by Richecoeour and Kyritsis [11] and Evans and Kyritsis [12] for rich flames propagating in curved ducts and by Pizza et al. [13] and Kurdyumov et al. [14] for lean flames propagating in a bidimensional micro-channel.

Some years ago, Lloyd and Weinberg [15] and Jones et al. [16] proposed the recirculation of heat as an alternative to extend the flammability limits in small-size burners. In this type of burners, the burned gas heat is recycled to preheat the unburned mixture, reducing the effect of the heat losses through the walls. In order to understand the dynamics of a flame with heat recirculation, Ronney [17], Ju and Choi [18] and Sánchez-Sanz [19] carried out theoretical studies in simplified geometries demonstrating, in particular, the extension of the flammability limits to leaner flames and the reduction of the minimum burner size that allows combustion as a function of the amount of heat recirculated.

As indicated previously, the use of liquid fuels as an alternative to batteries to power small-size devices falls on sufficiently high thermal efficiencies that have not been achieved yet. At least for rotary engines, this is, in part, due to the insufficient understanding of the effect of the fuel concentration in the incoming fresh mixture on the combustion process [3-5].
The brief review presented above has cited some works dealing with different aspects of small-scale combustion but, despite of its inherent practical importance, no research article could be found in the literature analyzing the effect of the equivalence ratio on the combustion of a premixed flame in a narrow channel. The present work attempts to cover that gap by undertaking a systematic investigation about the effect of the equivalence ratio on the dynamic of combustion in a bidimensional, micro-scale channel for fuels with different diffusivity.

Concretely, we aim to map the flame dynamics as a function of the mass flow rate, the equivalence ratio and the fuel Lewis number. With this idea in mind, the following section is dedicated to formulate the unsteady problem in non-dimensional form. After that, we'll solve numerically the steady version of these equations to obtain the steady-state solution in terms of the parameters of the problem. This steady solution will be used later to initiate an unsteady calculation that will provide the long-time behavior we need to catalog the flame dynamics. Simultaneously, we carry out a stability analysis in which we solve the linear eigenvalue problem obtained by perturbing the steady solution with a small-amplitude, time-dependent harmonic term. The appropriate combination of the results obtained from the unsteady computa-tion and from the stability analysis will be used to plot the stability maps that constitute the core of this work.

\section{Formulation}

We consider here a premixed combustible mixture flowing in a planar two-dimensional channel of height $h$ with a step-wise wall temperature that increases from the inlet value $T_{0}$ to a higher value $T_{w}$ at $x^{\prime}=0$, as sketched in Fig. 1 . In the thermo-diffusive approximation used here, the mixture density $\rho$, fuel $D_{F}$ and oxidizer $D_{O}$ molecular diffusivity, heat capacity $c_{p}$, and thermal diffusivity $D_{T}$ are defined as constants. The chemical reaction is modeled through an irreversible, single-step reaction of the form $v_{F} F+v_{O} O \rightarrow P+Q$ where $F, O, P$ and $Q$ denote the fuel, the oxidizer, the combustion products, and the heat released in the chemical reaction. Hereafter, $v_{F}$ and $v_{O}$ are the stoichiometric coefficient of the fuel and oxidizer, which have molecular weights $W_{F}$ and $W_{O}$, respectively. The equivalence ratio of the mixture is, therefore, defined as $\phi=$ $s Y_{F, 0} / Y_{O, 0}$ where $s=v_{O} W_{O} /\left(v_{F} W_{F}\right)$ is the mass of oxidizer needed to burn the unit mass of fuel, and $Y_{F, 0}$ and $Y_{O, 0}$ represent the mass fraction of fuel and oxidizer at $x \rightarrow-\infty$.

The molar fuel consumption rate is written in the form of an Arrhenius law $\omega=\rho^{2} \mathcal{A}\left(W_{F} W_{O}\right)^{-1} Y_{F}^{\prime} Y_{O}^{\prime} \exp \left(-E_{a} / R_{g} T\right)$, with $\mathcal{A}$ the pre-exponential factor, $E_{a}$ the activation energy, $R_{g}$ the universal gas constant, and $T$ is the local temperature of the mixture.

The system of differential equations describing the combustion problem introduced above, can be written in non-dimensional form by scaling the variables with the appropriate reference values. The species mass fractions are scaled using their inlet values $Y_{F, 0}$ and $Y_{O, 0}$, respectively, giving $Y_{F}=Y_{F}^{\prime} / Y_{F, 0}$ and $Y_{O}=Y_{O}^{\prime} / Y_{O, 0}$. The channel width $h$ and the diffusion time $h^{2} / D_{T}$ are chosen as the reference

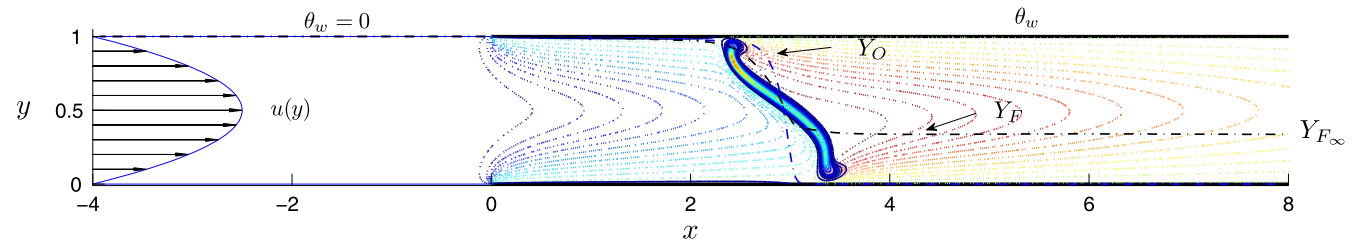

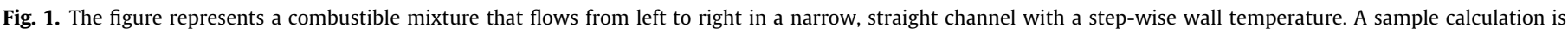

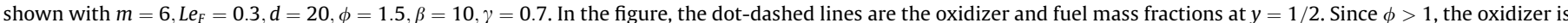

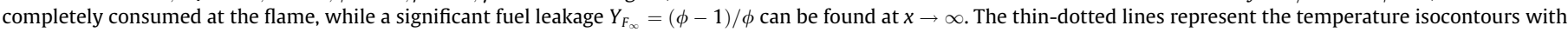
$\Delta \theta=0.05$, the solid-thick curves represent the reaction rate with $\Delta \omega=0.05$ and the dot-dashed curves are the mass fraction of fuel and oxidizer at $y=1 / 2$. 
units of length and time, defining the non-dimensional coordinates $x=x^{\prime} / h, y=y^{\prime} / h$ and time $t=t^{\prime} /\left(h^{2} / D_{T}\right)$. The non-dimensional temperature is defined as $\theta=\left(T-T_{0}\right) /\left(T_{s}-T_{0}\right)$, with $T_{0}$ the temperature of the incoming mixture and $T_{s}$ the adiabatic flame temperature of a mixture with an equivalence ratio $\phi$, so that

$T_{s}-T_{0}= \begin{cases}\frac{Y_{F, 0} Q}{c_{p} v_{F} W_{F}} & \phi<1 \\ \frac{Y_{O, 0} Q}{c_{p} v_{O} W_{O}} & \phi>1\end{cases}$

Introducing the non-dimensional variables defined above, the problem reduces to the integration of

$\frac{\partial \theta}{\partial t}+m \sqrt{d} u(y) \frac{\partial \theta}{\partial x}=\Delta \theta+d \Omega \frac{L e_{F}^{-1}}{1+\phi\left[-1+2 L e_{0} \beta^{-1}\right]}$

$\frac{\partial Y_{O}}{\partial t}+m \sqrt{d} u(y) \frac{\partial Y_{O}}{\partial x}=\frac{1}{L e_{O}} \Delta Y_{O}-d \Omega \frac{\phi L e_{F}^{-1}}{1+\phi\left[-1+2 L e_{O} \beta^{-1}\right]}$

$\frac{\partial Y_{F}}{\partial t}+m \sqrt{d} u(y) \frac{\partial Y_{F}}{\partial x}=\frac{1}{L e_{F}} \Delta Y_{F}-d \Omega \frac{L e_{F}^{-1}}{1+\phi\left[-1+2 L e_{O} \beta^{-1}\right]}$,

for lean flames and

$\frac{\partial \theta}{\partial t}+m \sqrt{d} u(y) \frac{\partial \theta}{\partial x}=\Delta \theta+d \Omega \frac{\phi L e_{0}^{-1}}{\phi-1+2 L e_{F} \beta^{-1}}$

$\frac{\partial Y_{O}}{\partial t}+m \sqrt{d} u(y) \frac{\partial Y_{O}}{\partial x}=\frac{1}{L e_{O}} \Delta Y_{O}-d \Omega \frac{\phi L e_{O}^{-1}}{\phi-1+2 L e_{F} \beta^{-1}}$

$\frac{\partial Y_{F}}{\partial t}+m \sqrt{d} u(y) \frac{\partial Y_{F}}{\partial x}=\frac{1}{L e_{F}} \Delta Y_{F}-d \Omega \frac{L e_{O}^{-1}}{\phi-1+2 L e_{F} \beta^{-1}}$,

for rich flames, where $m=U_{0} / S_{L}$ is the incoming mass flow rate, $S_{L}$ the planar flame speed and $U_{0}$ the mean flow velocity.

As shown above in Eq. (1), the maximum temperature depends on the mass fraction of the deficient species, that is completely consumed at the flame. The leakage of the excess reactant through the flame can be obtained by combining (3), (4), (6) and (7), to get the fuel and oxidizer mass fractions at infinity

$Y_{F_{\infty}}=0, \quad Y_{O_{\infty}}=1-\phi$ if $\phi<1$

$Y_{F_{\infty}}=\frac{\phi-1}{\phi}, \quad Y_{O_{\infty}}=0$ if $\phi>1$

The system of equations simplifies for lean and rich flames, reducing the problem to the integration of the equation of energy and the equation for the deficient species mass fraction. Indeed, if $\phi \rightarrow 0$, the oxidizer mass fraction remains constant and the problem reduces to that solved by Kurdyumov et al. in [14] in the equidiffusional case $L e_{O}=L e_{F}=1$.

In non-dimensional form, the combustion rate is written as

$\Omega=k(\theta) Y_{F} Y_{O}$ and $k(\theta)=\frac{\beta^{2}}{2 u_{p}^{2}} \exp \left[\frac{\beta(\theta-1)}{1+\gamma(\theta-1)}\right]$,

with the value of the heat release parameter $\gamma=\left(T_{s}-T_{0}\right) / T_{s}=0.7$, the Zeld'ovich number $\beta=E\left(T_{s}-T_{0}\right) / R_{g} T_{s}^{2}=10$ and the Damköler number $d=h^{2} / \delta_{T}^{2}=20$ kept fixed hereafter, with $\delta_{T}=D_{T} / S_{L}$ representing the thermal flame thickness. In Eqs. (2)-(4), $\Delta=\partial^{2} / \partial_{x x}+$ $\partial^{2} / \partial_{y y}$ is the Laplacian operator and $u(y)=6 y(1-y)$ is the Poiseuille velocity profile.

Above equations are integrated by imposing boundary conditions upstream $x \rightarrow-\infty$ for both temperature and mass fractions

$\theta=Y_{F}-1=Y_{O}-1=0$,

while far downstream $x \rightarrow \infty$ we impose

$\frac{\partial \theta}{\partial x}=\frac{\partial Y_{F}}{\partial x}=\frac{\partial Y_{O}}{\partial x}=0$.
The physical domain is truncated to a finite computational domain $x \in\left[\begin{array}{ll}x_{\min } & x_{\max }\end{array}\right]$, where $x_{\min }$ and $x_{\max }$ must be carefully chosen in order to assure the fulfillment of the boundary conditions. Typically $x_{\min }=-10$ and $x_{\max }=10$ represent a sufficiently long computational domain even for very diffusive fuels and large mass flow rates.

The boundary conditions at the non-porous walls are given by

$\theta-\theta_{w} \Theta(x)=\frac{\partial Y_{F}}{\partial y}=\frac{\partial Y_{O}}{\partial y}=0$ at $y=0$ and $y=1$

where $\Theta(x)$ is the Heaviside step function and $\theta_{w}=0.6$ the wall temperature.

The factor $u_{p}=S_{L} / U_{L}$ introduced above in Eq. (8), represents the ratio between the planar flame speed $S_{L}$ obtained with finite Z'eldovich number $\beta$ and the planar flame speed $U_{L}$ obtained with infinite Z'eldovich number $\beta \rightarrow \infty$ for a mixture with equivalence ratio $\phi[20]$,

$U_{L}^{2}=2 \mathcal{A} \rho D_{T} S Y_{F, 0} \frac{v_{F} L e_{F}}{W_{O} \beta^{2}}\left(\phi^{-1}-1+2 \frac{L e_{O}}{\beta}\right) \exp \left[-\frac{E_{a}}{R_{g} T_{s}}\right] \quad \phi \leqslant 1$
$U_{L}^{2}=2 \mathcal{A} \rho D_{T} S^{-1} Y_{O, 0} \frac{v_{O} L e_{O}}{W_{F} \beta^{2}}\left(\phi-1+2 \frac{L e_{F}}{\beta}\right) \exp \left[-\frac{E_{a}}{R_{g} T_{s}}\right] \quad \phi \geqslant 1$

The factor $u_{p}$ ensures that, for given $\beta$ and $\phi$, the non-dimensional flame speed for a planar adiabatic flame equals one. The adequate calculation of the factor $u_{p}$ requires the resolution of the following one-dimensional eigenvalue problem

$$
\begin{aligned}
& \phi \leqslant 1\left\{\begin{array}{l}
\frac{d \theta}{d \xi}=\frac{d^{2} \theta}{d \xi^{2}}+\Omega \frac{L e_{F}^{-1}}{1+\phi\left[-1+2 L e_{O} \beta^{-1}\right]} \\
\frac{d Y_{O}}{d \xi}=L e_{O}^{-1} \frac{d^{2} Y_{O}}{d \xi^{2}}-\Omega \frac{\phi L e_{F}^{-1}}{1+\phi\left[-1+2 L e_{O} \beta^{-1}\right]} \\
\frac{d Y_{F}}{d \xi}=L e_{F}^{-1} \frac{d^{2} Y_{F}}{d \xi^{2}}-\Omega \frac{L e_{F}^{-1}}{1+\phi\left[-1+2 L e_{O} \beta^{-1}\right]}
\end{array}\right. \\
& \phi \geqslant 1\left\{\begin{array}{l}
\frac{d \theta}{d \xi}=\frac{d^{2} \theta}{d \xi^{2}}+\Omega \frac{\phi L e_{O}^{-1}}{\phi-1+2 L e_{F} \beta^{-1}} \\
\frac{d Y_{O}}{d \xi}=L e_{O}^{-1} \frac{d^{2} Y_{O}}{d \xi^{2}}-\Omega \frac{\phi L e_{O}^{-1}}{\phi-1+2 L e_{F} \beta^{-1}} \\
\frac{d Y_{F}}{d \xi}=L e_{F}^{-1} \frac{d^{2} Y_{F}}{d \xi^{2}}-\Omega \frac{L e_{O}^{-1}}{\phi-1+2 L e_{F} \beta^{-1}}
\end{array}\right.
\end{aligned}
$$

where $\xi=x^{\prime} /\left(D_{T} / S_{L}\right)$. A standard shooting method has been used to solve the above system of equations with boundary conditions $\theta=Y_{F}-1=Y_{O}-1=0$ at $\xi \rightarrow-\infty$ and $\theta-1=d Y_{F} / d \xi=d Y_{O} / d \xi=$ 0 a t $\xi \rightarrow \infty$, giving the evolution of $u_{p}$ with the fuel Lewis number that has been plotted in Fig. 2 for different equivalence ratios.

\section{The stationary solution}

To analyze the effect of the stoichiometry on the stability of a premixed flames, we need first to compute the base flow by integrating the steady version of Eqs. (2)-(7). To do so, we used a thirdorder, four-point finite difference scheme in both $x$ and $y$ direction with $\Delta x=\Delta y=1 \times 10^{-2}$. A Gauss-Seidel method with over relaxation was used to solve the system in a finite domain $-x_{\max }<x$ $<x_{\max }, 0<y<1$, where it is required sufficiently large values of $x_{\max }$ to make the solution independent of the computa-tional domain. The iteration procedure continues until the differ-ence between the solutions in two consecutive iterations was below $1 \times 10^{-4}$.

Following [14], we characterize the solution by calculating the position $x_{f}$ and the symmetry of the flame by means of the parameter $S$, defined below in Eq. (16). The location of the flame is unequivocally determined by looking for the axial location at which the reaction attains its maximum value at $y=1 / 2, \Omega\left(x_{f}\right)$ 


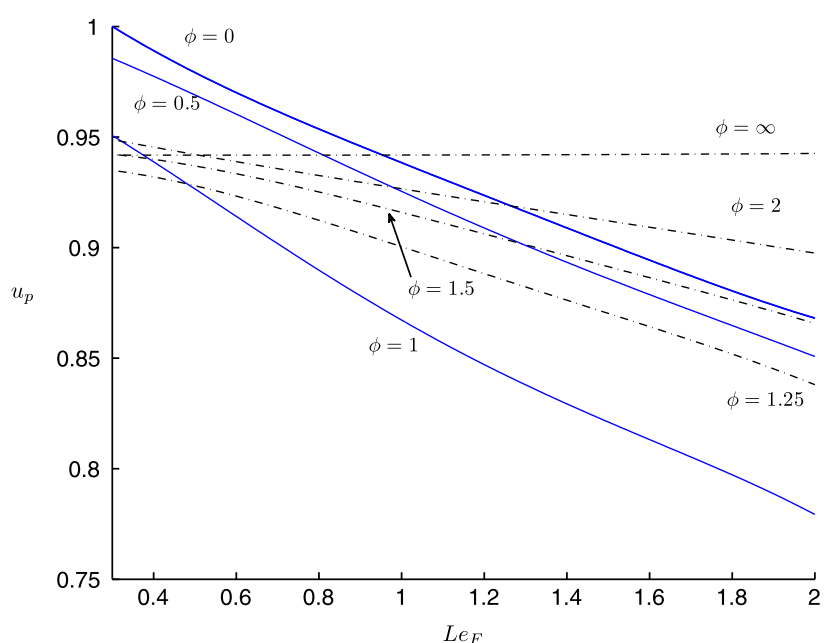

Fig. 2. Numerical values of factor $u_{p}=S_{L} / U_{L}$ obtained from the integration of (15) or (14) with $L e_{O}=1, \beta=10$ and $\gamma=0.7$.

$=\max (\Omega(x, y=0.5))$. As anticipated above, we evaluate the flame symmetry by means of the symmetry parameter $S$, defined as:

$S=\int_{-\infty}^{\infty}\left[\int_{0}^{1 / 2}\{\theta(x, y)-\theta(x, 1-y)\} d y\right] d x$

Since this parameter is equal to zero if the flame remains symmetrical and becomes non-zero when the symmetry of the flame is broken, we use $S$ to quantify the level of symmetry of the flame.

To check the accuracy of the numerical scheme, we have compared the evolution of the flame location $x_{f}$ for different mass flow rates $m$ in the limit of very lean mixtures $\phi=0$ for $L e_{F}=1$, with the results obtained by Kurdyumov et al. [14]. The match between the results is almost perfect except for small values of the mass flow rate $m$ where small discrepancies in the flame location $x_{f}$ were observed, probably due to a excessively short computational domain in the Kurdyumov et al.'s computations: they fixed the upstream border at $x=-3$ instead of at $x=-10$, as we did in this work.

In Fig. 3 we plot the flame location $x_{f}$, the flame temperature at the mid-plane $T_{f}=\theta\left(x_{f}, y=1 / 2\right)$ and the symmetry parameter $S$ as a function of the incoming mass flow rate $m$ for a very diffusive fuel with Lewis numbers $L e_{F}=0.3$, such as hydrogen. In this case, the solution remains unique and symmetric only for small values of $m$, bifurcating at $m=m_{c}=(1.05,1.15,3.25)$ for $\phi=(0,0.5,1.5)$. For $m>m_{c}$ three different solutions are found: one symmetric $S=0$ and two asymmetric $(S>0$ or $S<0)$. Notice that the multiplicity of solutions disappears completely in near-stoichiometric mixtures $\phi \simeq 1$, when only a symmetric solution $S=0$ could be found. The stability of the solution branches found above will be studied in the following sections by means of both a stability analysis and an unsteady computation.

The picture depicted above changes when less diffusive fuels are considered, as can be seen in Fig. 4 where the flame temperature $T_{f}$ and location $x_{f}$ are plotted for fuels with Lewis number $L e_{F}=1$ and $L e_{F}=1.4$, such as, for example, methane and propane respectively. In both cases, the steady-state solution remains unique and symmetric $S=0$ for all the mass fractions considered. A similar behavior has been reported before by Kurdyumov in [21], where very lean flames propagating in an adiabatic channel were studied.

\section{The unsteady calculations}

To study the flame dynamics for $t \rightarrow \infty$, we have integrated numerically the system of Eqs. (2)-(7). in the entire domain $0 \leqslant y \leqslant 1, x_{\min }<x<x_{\max }$ by using a second order numerical scheme in both time and space with time step $\Delta t=10^{-5}$ and grid spacing $\Delta x=\Delta y=10^{-2}$. To initiate the unsteady computation, we have used two different initial conditions to test its impact on the final solution. In first place, we used the stationary solution computed before in Section 3. During the first time-steps of the computation, we introduced a small perturbation in the mass fraction to trigger the instability in the form

$\tilde{m}=m+d m$,

to later impose $\tilde{m}=m$. If, for a given set of parameters, the steady solution turned out to be stable, the perturbation smooths out after a short period of time and the stationary solution is recovered. Otherwise, the perturbation grows and develops the corresponding oscillatory behavior, as depicted in Fig. 7. As indicated in the following sections, we have varied the amplitude of the perturbation in the range $d m / m=[0-0.5]$ to check that the solution does not depend on the perturbation amplitude $d m$.

Alternatively, we used an initial condition in which we set a uniform distribution for the mass fraction of fuel and oxidizer $Y_{D}=Y_{E}=1$, and a hot-spot temperature distribution in the form

$\theta=\theta_{s} \exp \left\{-\left[\left(x-x_{s}\right)^{2}+\left(y-y_{s}\right)^{2}\right] / r_{s}^{2}\right\}$

where the location of the hot spot $\left(x_{s}, y_{s}\right)$, its radius $r_{s}$ and its maximum temperature $\theta_{s}$ were varied to check the sensitivity of the final solution with respect to their value. Representative values of theses parameters are $x_{s}=0.5, y_{s}=0.2, r_{s}=0.5$ and $\theta_{s}=0.95$. An example of the evolution of the isocontours of temperature and reaction rate using the initial condition (18) can be seen in Fig. 5 .

\section{Stability analysis}

In parallel to the unsteady calculation described above, we have carried out a linear stability analysis of the stationary flames computed above in Section 3. To do so, we have introduced a small harmonic perturbation on the form

$\theta(x, y, t)=\theta^{0}(x, y)+\varepsilon \theta^{1}(x, y) e^{\lambda t}$
$Y_{F}(x, y, t)=Y_{F}^{0}(x, y)+\varepsilon Y_{F}^{1}(x, y) e^{\lambda t}$
$Y_{O}(x, y, t)=Y_{O}^{0}(x, y)+\varepsilon Y_{O}^{1}(x, y) e^{\lambda t}$

where $\lambda=\lambda_{r}+i \lambda_{i}, \lambda_{r}=\operatorname{Re}\{\lambda\}, \lambda_{i}=\operatorname{Im}\{\lambda\}, i=\sqrt{-1}$ and the superindex 0 is used to denote the stationary solution obtained above. The substitution of these expressions into (2)-(7) leads to the linearized problem

$\phi \leqslant 1\left\{\begin{array}{l}\lambda \theta^{1}=-\sqrt{d} m u(y) \frac{\partial \theta^{1}}{\partial x}+\Delta \theta^{1}+d k\left(\theta^{0}\right)\left(A \theta^{1}+Y_{O}^{0} Y_{F}^{1}+Y_{O}^{1} Y_{F}^{0}\right) \\ \frac{L e_{F}^{-1}}{1+\phi\left[-1+2 L e_{O} \beta^{-1}\right]} \\ \lambda Y_{O}^{1}=-\sqrt{d} m u(y) \frac{\partial Y_{O}^{1}}{\partial x}+\frac{1}{L e_{0}} \Delta Y_{O}^{1}-d k\left(\theta^{0}\right)\left(A \theta^{1}+Y_{O}^{0} Y_{F}^{1}+Y_{O}^{1} Y_{F}^{0}\right) \\ \frac{\phi L e_{F}^{-1}}{1+\phi\left[-1+2 L e_{O} \beta^{-1}\right]} \\ \lambda Y_{F}^{1}=-\sqrt{d} m u(y) \frac{\partial Y_{F}^{1}}{\partial x}+\frac{1}{L e_{F}} \Delta Y_{F}^{1}-d k\left(\theta^{0}\right)\left(A \theta^{1}+Y_{O}^{0} Y_{F}^{1}+Y_{O}^{1} Y_{F}^{0}\right) \\ \frac{L e_{F}^{-1}}{1+\phi\left[-1+2 L e_{O} \beta^{-1}\right]}\end{array}\right.$

$\phi \geqslant 1\left\{\begin{array}{l}\lambda \theta^{1}=-\sqrt{d} m u(y) \frac{\partial \theta^{1}}{\partial x}+\Delta \theta^{1}+d k\left(\theta^{0}\right)\left(A \theta^{1}+Y_{O}^{0} Y_{F}^{1}+Y_{O}^{1} Y_{F}^{0}\right) \\ \frac{\phi L_{O}^{-1}}{\phi-1+2 L e_{F} \beta^{-1}} \\ \lambda Y_{O}^{1}=-\sqrt{d} m u(y) \frac{\partial Y_{O}^{1}}{\partial x}+\frac{1}{L e_{0}} \Delta Y_{O}^{1}-d k\left(\theta^{0}\right)\left(A \theta^{1}+Y_{O}^{0} Y_{F}^{1}+Y_{O}^{1} Y_{F}^{0}\right) \\ \frac{\phi L e_{O}^{-1}}{\phi-1+2 L e_{F} \beta^{-1}} \\ \lambda Y_{F}^{1}=-\sqrt{d} m u(y) \frac{\partial Y_{F}^{1}}{\partial x}+\frac{1}{L e_{F}} \Delta Y_{F}^{1}-d k\left(\theta^{0}\right)\left(A \theta^{1}+Y_{O}^{0} Y_{F}^{1}+Y_{O}^{1} Y_{F}^{0}\right) \\ \frac{L e_{O}^{-1}}{\phi-1+2 L e_{F} \beta^{-1}}\end{array}\right.$ 

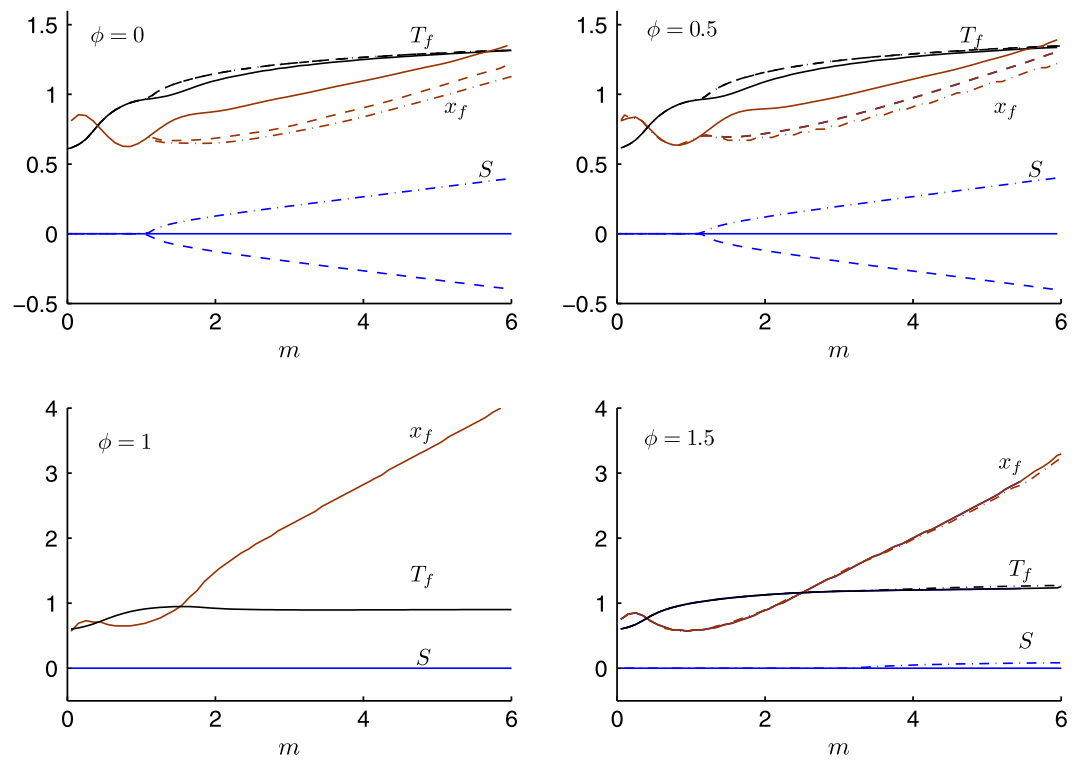

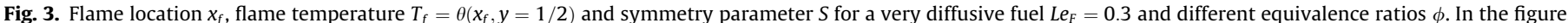

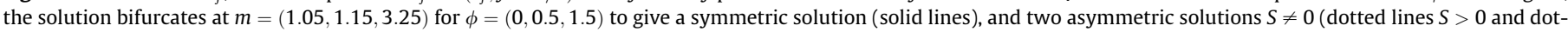
dashed lines $S<0$ ). For stoichiometric mixtures $\phi=1$, the solution is unique and symmetric.
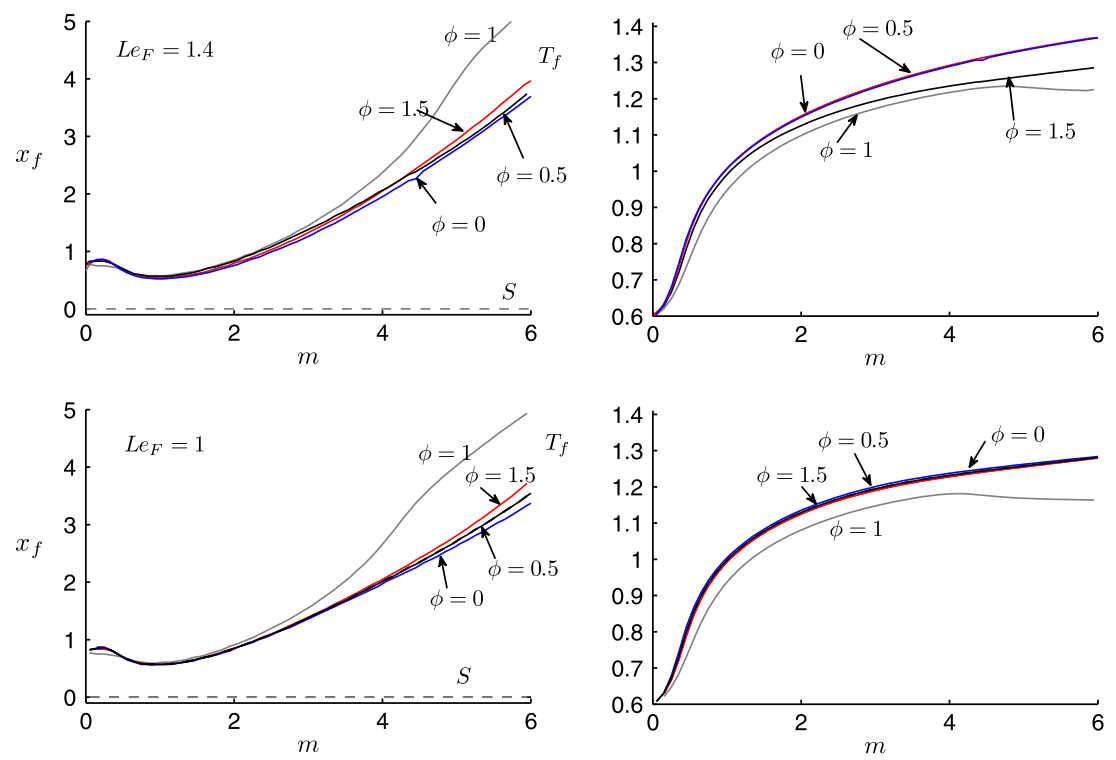

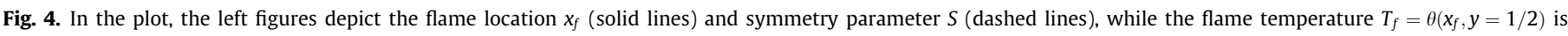

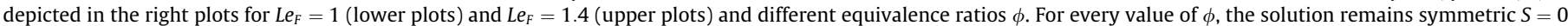
and unique.

where $A=\frac{\beta}{\left[1+\gamma\left(\theta^{0}-1\right)\right]^{2}} Y_{O}^{0} Y_{F}^{0}$. The solution of the above system determines, in particular, the value of the eigenvalue $\lambda$ whose real part $\lambda_{r}$ represents the growth rate of the perturbation, initially of or$\operatorname{der} O(\varepsilon) \ll 1$.

The multi-dimensional structure of the steady states makes the calculation of the whole spectrum of eigenvalues very expensive from a computational point of view. Nevertheless, to determine the linear stability of a steady-state, we only need information about the eigenvalue with the largest real part $\lambda_{r}$, so that $\lambda_{r}>0$ and $\lambda_{r}<0$ would indicate unstable and stable solutions, respectively. Notice, anyway, that this analysis does not provide any information about the time evolution of the flame once the instability has emerged.
For the reasons exposed above, we used the method developed by Kurdyumov in [21] to compute the eigenvalue with the largest real part. To study the emergence of the symmetric and asymmetric modes, we solved the above set of equations in the half-height domain $0 \leqslant y \leqslant 1 / 2$ with boundary conditions

$\frac{\partial \theta^{1}}{\partial y}=\frac{\partial Y_{F}^{1}}{\partial y}=\frac{\partial Y_{O}^{1}}{\partial y}=0$ at $y=1 / 2$ and $\theta^{1}=\frac{\partial Y_{F}^{1}}{\partial y}=\frac{\partial Y_{O}^{1}}{\partial y}=0$ at $y=0$

for the symmetric modes. Alternatively, for the non-symmetric modes, we impose the boundary conditions

$\theta^{1}=Y_{F}^{1}=Y_{O}^{1}=0$ at $y=1 / 2$ and $\theta^{1}=\frac{\partial Y_{F}^{1}}{\partial y}=\frac{\partial Y_{O}^{1}}{\partial y}=0$ at $y=0$. 


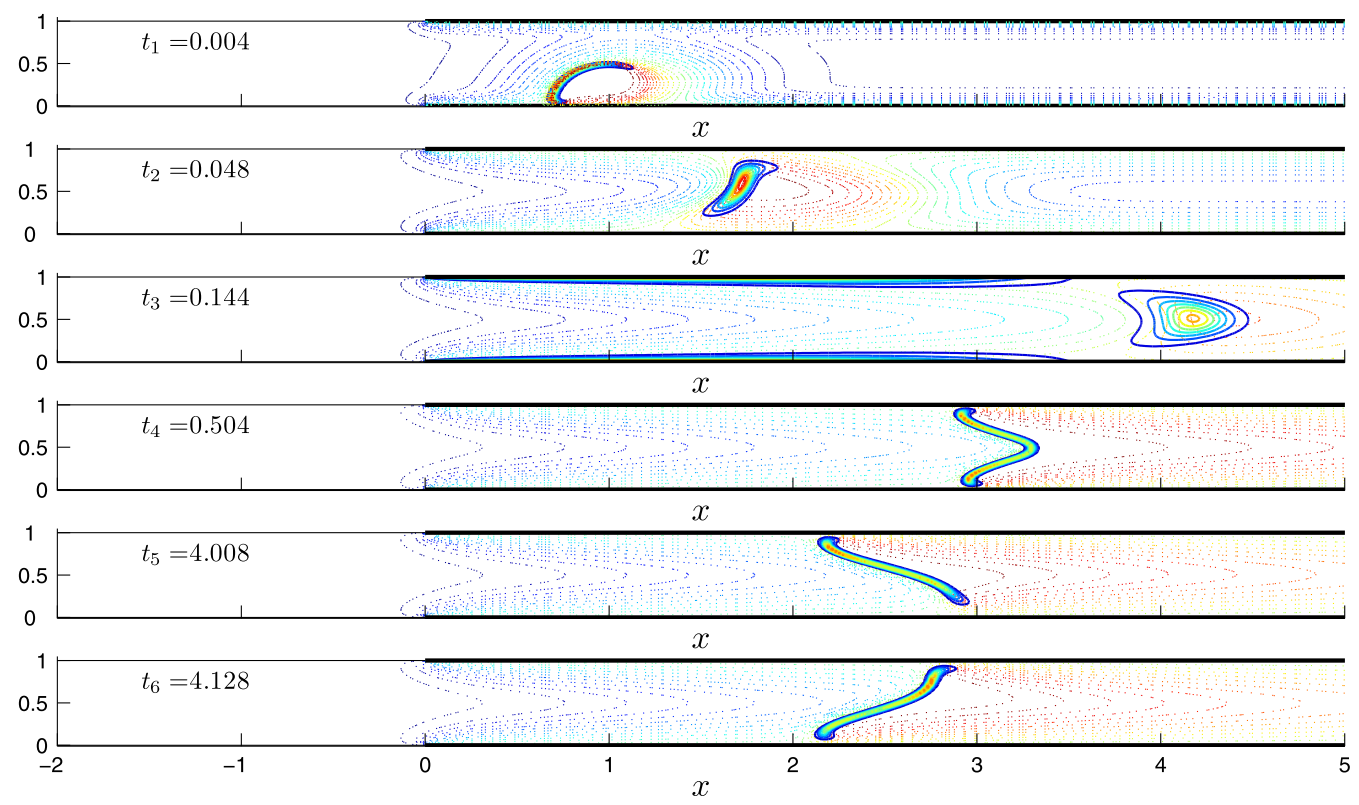

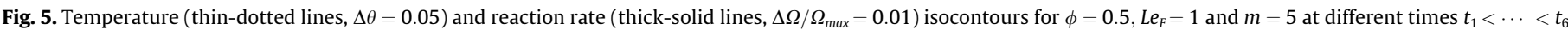

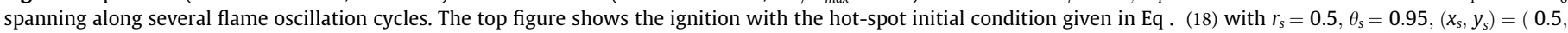

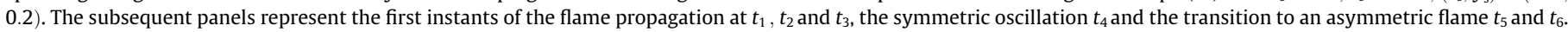
In the figure, $\Omega_{\max }=[38.499,3.354,0.224,23.025,16.966,16.671]$ for $t=[0.004,0.048,0.144,0.504,4.008,4.128]$, respectively.

\subsection{Very diffusive fuels $L e_{F}=0.3$}

For very diffusive fuels, the existence of multiple steady-state solutions, shown above in Section 3, anticipates the great variety of stability behaviors depicted in the stability map that constitutes Fig. 6. In this plot, we analyze the behavior for $t \rightarrow \infty$ of each of the steady solutions in the incoming mass flow rate $m$ vs. the equivalence ratio $\phi$ parametric space, indicating the regions in which the response of the steady solution to a small perturbation is coincident. To build this figure, we have carried out a series of unsteady calculations using, as initial condition, all the possible stationary solutions reported above in Section 3 for each value of $m$ and $\phi$. For instance, to determine the flame behavior for $t \rightarrow \infty$ in the case $\phi=0.5, m=4$, we initiated the computation by using the symmetric stationary solution first $S=0$ and we waited a sufficiently long time to determine the final flame behavior. The same calculation was repeated later but using the asymmetric solutions $S>0$ and $S<0$ as the initial condition of the successive calculations. The respective long-time flame behaviors were later compared to analyzed possible coincidences.

Also, in Fig. 7 we depicted the evolution with time of the flame position $x_{f}$ and of the symmetry parameter $S$, for $\phi=0.5$ and a set of representative mass fractions (indicated with symbols in Fig. 6). Each region of the stability map is identified with initials that distinguish the behavior of the solution at $t \rightarrow \infty$. Their meaning is explained next:

$\mathrm{St}_{a}, \mathrm{~S}{ }_{s} \mathrm{t} \rightarrow$ A steady, symmetric or asymmetric flame is achieved when the initial condition is symmetric or asymmetric respectively. Both solutions can coexist (Fig. 7d). $\mathrm{St}_{a}, \mathrm{U}_{s} \rightarrow$ The asymmetric solutions remain stable with the value of the symmetry parameter $S$ equal to that of the initial solution. On the other hand, a pulsating mode develops when the symmetric solution losses its stability and initiates a symmetric $S=0$, periodical oscillating. Both solutions can coexist (Fig. 7c).

$\mathrm{St}_{a} \rightarrow$ The solution evolves towards a steady asymmetric solution independently of the initial condition used to start the unsteady calculation (Fig. 7b).

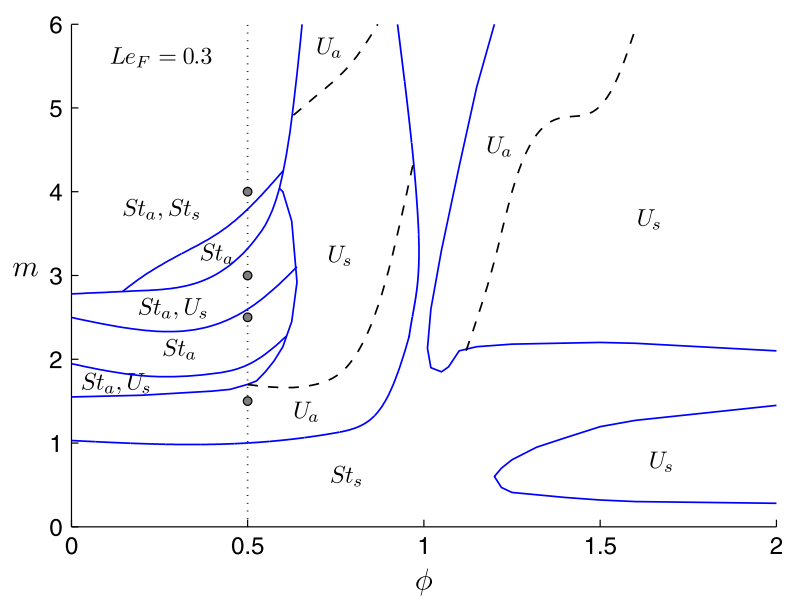

Fig. 6. Stability map delineating the regions with different flame behavior in the $m$ vs. $\phi$ space for $L e_{F}=0.3$. Each region is labeled with initials whose meaning is described in the main text. The symbols $\bullet$, located at $\phi=0.5$, indicate a set of representative mass fractions whose evolution with time has been plotted in Fig. 7

$\mathrm{St}_{s} \rightarrow$ The solution evolves towards a steady symmetric solution independently of the initial condition used to start the unsteady calculation.

$\mathrm{U}_{a} \rightarrow$ The flame oscillates periodically with $S \neq 0$ independently of the initial condition used (Fig. 7a).

$\mathrm{U}_{s} \rightarrow$ The flame oscillates periodically with $S=0$ independently of the initial condition used.

Additionally, in a thin region located around the dashed lines included in Fig. 6, the flame always oscillates periodically but the shape of the flame remains symmetric $S=0$ only if the initial condition is symmetrical while becomes asymmetric $S \neq 0$ when a non-symmetric initial condition is used. A similar behavior was reported by [14], where two oscillatory solutions are found to coexist.

The map plotted in Fig. 6 distinguishes, clearly, between the behavior of rich and lean premixed flames, as expected due to 


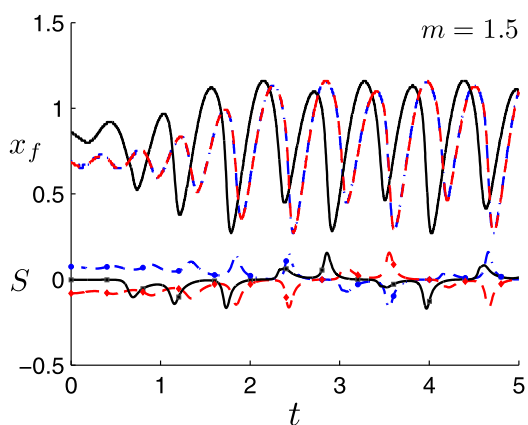

(a)

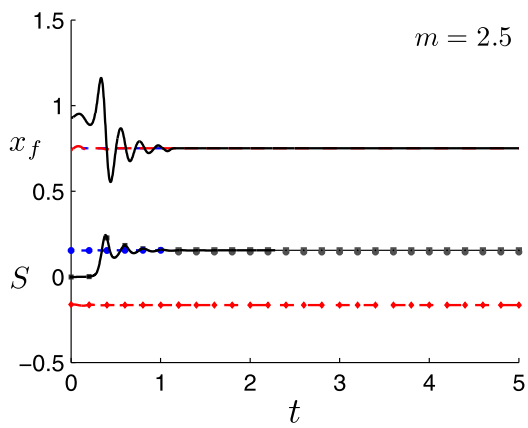

(b)

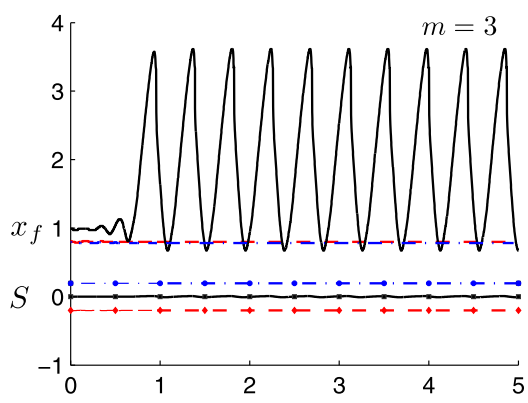

(c)

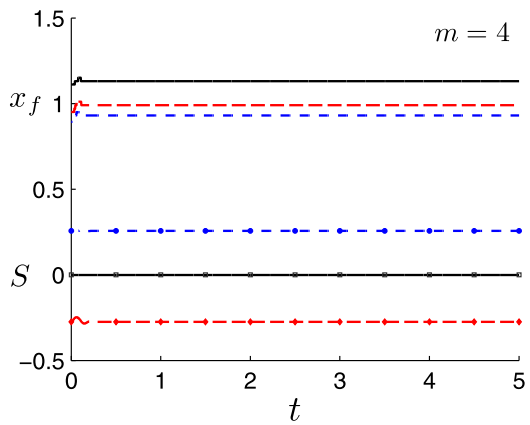

(d)

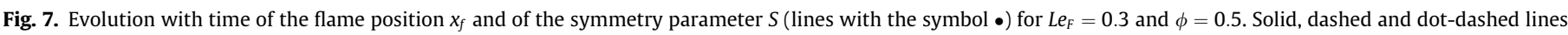

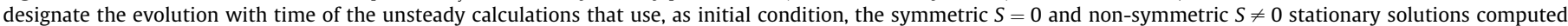
previously.

the strong difference between the Lewis numbers of fuel and 2oxidizer. Very lean flames $\phi<0.6$ are stable for most of the mass flow rates considered, leaving apart the interval $m \in$ [11.5], when the flame oscillates asymmetrically independently of the initial condition used to start the computation, and $m \in[1.54]$, when the flame behavior depends on the initial condition. In the range $0.6<\phi<1$ the flame oscillates for every mass flow rate above $m \gtrsim 1$ while, remarkably, around $\phi=1$ the stationary solution is stable for every $m$.

The behavior for rich flames $\phi>1$ is markedly different. The region of instability extends to occupy the whole region $m>2$ for $\phi>1.05$, approximately. The flame symmetry changes with the mass flow rate $m$ and the stoichiometric ratio $\phi$. Even though the solution is unsteady in both cases, the asymmetric oscillation, with alternative changes in the sign of $S$, is confined to values of $\phi<1.6$ and increasing mass flow rates. Contrarily, symmetric oscillations $S=0$ are expected to emerge for larger $\phi$ and mass flow rates in the intervals $m \in[0.21]$ and $m \in[2 \infty]$.

The flame behavior described above in Fig. 6 is backed up by the analysis of the eigenvalues plotted in Fig. 8, obtained from the linear stability analysis described above in Section 5 in the case $\phi=$ 0.5 using the symmetric stationary solution as the base profile. According to that figure, approximately for $m \in[1.13 .3]$ the real part of the eigenvalues of the non-symmetric modes are positive $\lambda_{r}$ $>0$ with $\lambda_{i}=0$ while for $m \in$ [1.353.2] the real part of the eigenvalue $\lambda_{r}>0$ with $\lambda_{i} \neq 0$, suggesting the onset of an instability that evolves the solution towards an oscillatory, symmetric behavior or towards an asymmetric $S \neq 0$, stationary solution. Below and above the interval mass flow rates indicated above, $\lambda_{r}<0$ and both the symmetric and non-symmetric modes are stable. Notice that the linear stability analysis does not predict which of these two possibles solutions will end up developing. In this and other similar cases treated in this work, we have solved the uncertainty by means of a time dependent calculation, as that shown in Fig. 7, that

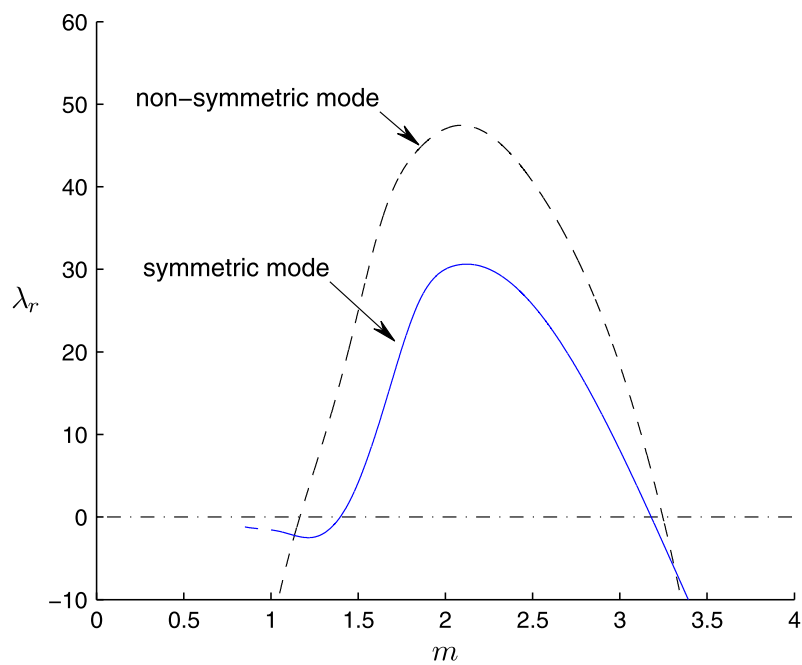

Fig. 8. Real eigenvalues $\lambda_{r}$ for the symmetric and non-symmetric modes vs. $m$ for $L e_{F}=0.3$ and $\phi=0.5$. The curve is plotted with solid line when $\lambda_{i} \neq 0$ while dashed lines are used when $\lambda_{i}=0$.

settles which solution will prevail over the other at $t \rightarrow \infty$ for a given initial condition.

Notice that a similar stability analysis could have been carried out by using the non-symmetric stationary solution as the base profile. Similar results are expected to be obtained, with the real part of the eigenvalue becoming positive $\lambda_{r}>0$ with $\lambda_{i} \neq 0$ approximately in the range $m \in[11.6]$ for $\phi=0.5$.

The flame dynamics, even in the simplified problem considered here, is rich and complex, as can be seen in Fig. 9. Specifically, in this figure we plot the time evolution of the temperature and reaction rate isocontours obtained from the integration of Eqs. (2)-(4) 
for $\phi=0.5, L e_{F}=0.3$ and $m=1.5$, using the symmetric steady solution as initial condition. Notice that, in this case, the three stationary solutions obtained from the integration of the steady version of the conservation equations are unstable, and they all present similar long-time evolutions of flame location $x_{f}$ and symmetry parameter $S$, as was anticipated above in Fig. 7a). The sequence of solutions starts at $t=t_{1}=13.4$, when the flame is almost symmetrical and the maximum temperature is achieved at the center of the channel $y=1 / 2$. At $t=t_{2}$, the symmetry of the flame is progressively lost, with the parameter $S>0$ achieving a local maximum at $t=t_{3}=14.1$, when the flame is partially extinguished in the upper part of the channel $y>1 / 2$ and the flame temperature at $y=1 / 2$ is well above unity $T_{f}=1.219$. At $t=t_{4}$, the flame reignites and almost reaches both walls of the channel, where the heat losses are sufficiently large to locally quench the flame. Finally, at $t=t_{5}$ the flame acquires a form that is almost the specular reflection of that shown in previous panels, with the symmetry parameter $S$ moving towards a local minimum and a reaction rate in the lower part of the channel so small that the flame can be considered quenched.

Notice that the flame behavior described here using the thermo-diffusive approximation reproduces qualitatively well the results reported by Pizza et al. in [13] in their full-chemistry, direct numerical simulation of lean $\phi=0.5$ hydrogen premixed flames propagating in a microchannel.

\subsection{The equidiffusional case Le $_{F}=1$}

In the equidiffusional case, both fuel and oxidizer have the same Lewis number $L e_{O}=L e_{F}=1$. In this case, the governing equations are symmetrical with respect to $\phi=1$, as can be easily checked by looking at Eqs. (2)-(7). Correspondingly, we expect the same response to a perturbation for lean $\phi<1$ and rich mixtures $\phi>1$. This point is confirmed in Fig. 10, where we depict, in a $m$ vs. $\phi$ plot, the stability behavior of the stationary solutions calculated above in Section 3.

As before, the stationary solution obtained for very small and very large mass flow rates $m$ remains stable and symmetric (recall that, according to Fig. 4, the steady solution is always unique and symmetric for $L e_{F}=1$ ). Also, as we approach stoichiometric mixtures $\phi \simeq 1$, the flame becomes more stable and the instability region gets confined to an interval of mass flow rates $m \in[0.61 .15]$ at which a symmetric, oscillatory instability emerges. To strengthen this result, we have plotted in Fig. 16 the real part of the eigenvalue $\lambda_{r}$ as a function of $m$ for $\phi=1$. As expected, $\lambda_{r}>0$ only in a narrow gap of mass flow rates $m$ that coincides with the values obtained above from the unsteady calculation. A similar result was reported by Maruta et al. [9] in their experiments in a narrow duct with methane/air mixtures near $\phi=1$.

At moderately large mass flow rate, the unsteady computations shows an unexpected behavior. Shortly after the beginning of the calculation, the flame initiates a symmetric oscillation that seems to saturate at a constant amplitude. After a relatively long time, a new transition is observed in the solution, that evolves towards an oscillation with lower amplitude and significantly higher frequency. The symmetry of the flame is then broken, evolving periodically between positive and negative values of the symmetry parameter $S$. An example of this behavior can be seen in Fig. 10 for $\phi=0.5$ and $m=5$. This transition is not observed for smaller mass flow rates, when the symmetric oscillation persists, at least, up to $t=100$ when the unsteady computation was stopped.

The analysis of the eigenvalues in this case does not anticipate the emergence of the asymmetric oscillations. In Fig. 11 we plot the real part of the eigenvalue $\lambda_{r}$ for the symmetric and asymmetric modes as a function of the mass flow rate $m$ for $\phi=0.5$. As can be seen in this figure, both symmetric and asymmetric modes become unstable almost at the same mass flow rate, and their evolution with $m$ is almost identical. As we indicated above, the simple stability analysis carried out here cannot anticipate the final flame behavior and it is the unsteady calculation what will reveal the actual behavior.

In [14], Kurdyumov et al. gave account of a similar behavior. In this case, the asymmetric oscillation arises, in the limit $\phi \rightarrow 0$, only for certain initial conditions. To check the influence of the initial condition on the transition from symmetric to asymmetric flames we have tested several initial conditions to start the unsteady computation. In the first place, we initiated the calculation using the stationary solution obtained in Section 3 but imposing different

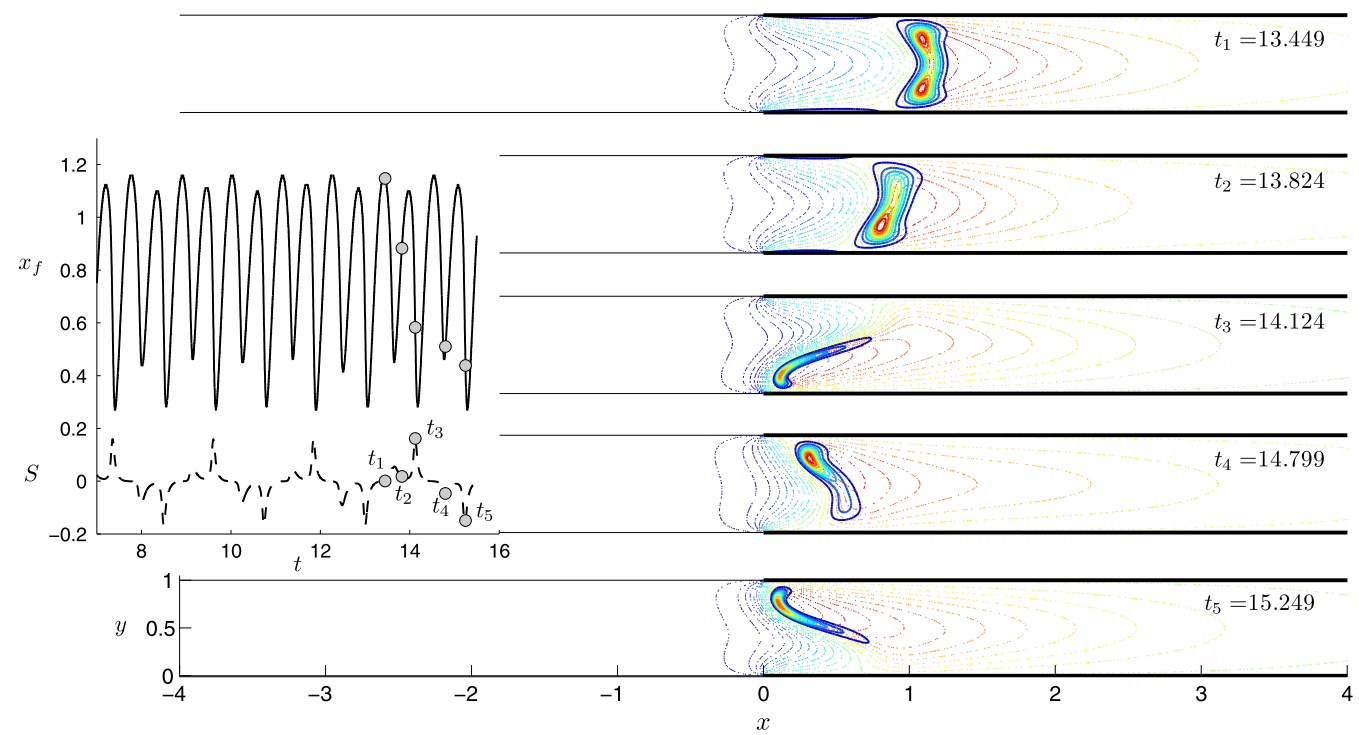

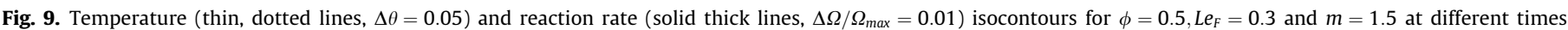

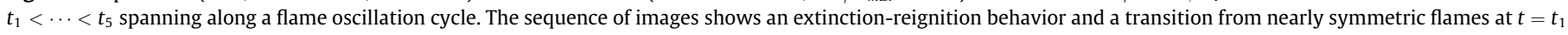

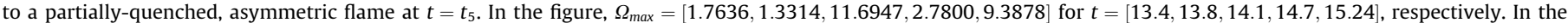

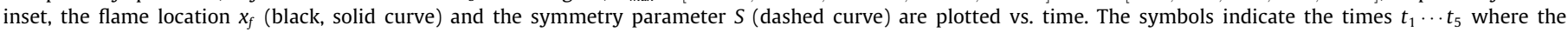
isocontours are plotted in the main figure. 


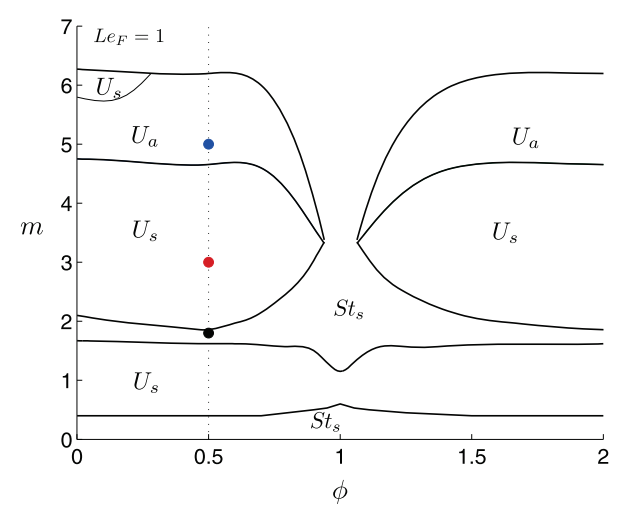

(a)

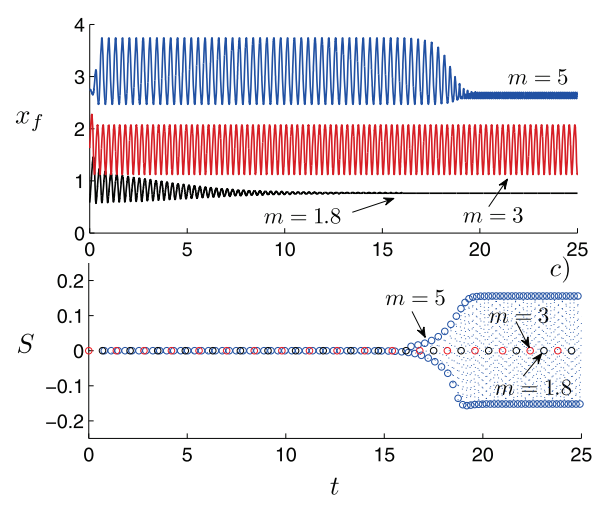

(b)

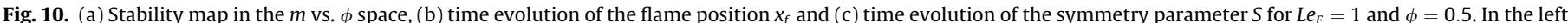

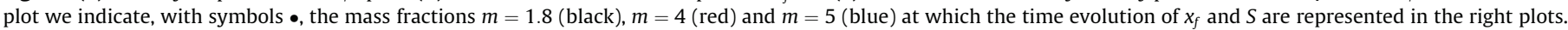
(For interpretation of the references to color in this figure legend, the reader is referred to the web version of this article.)

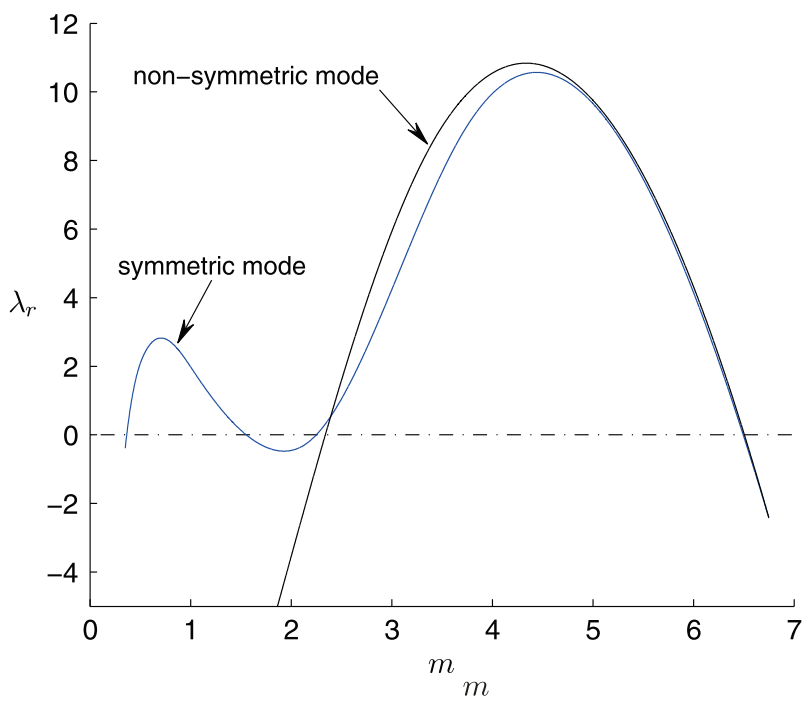

Fig. 11. Real eigenvalue $\lambda_{r}$ for the symmetric (solid lines) and non-symmetric modes (dashed line) vs. $m$ for $L e_{F}=1$ and $\phi=0.5$. In the whole range of mass flow rates, $\lambda_{i} \neq 0$.

perturbation amplitudes $d m / m=(0,0.1,0.2,0.5)$ in Eq. (17). In the second place, we have used the hot-spot initial condition given in Eq. (18), with $r_{s}=0.5, \theta_{s}=0.95$ and $\left(x_{s}, y_{s}\right)=(0.5,0.2)$ and $\left(x_{s}, y_{s}\right)=(0.5,0.5)$. In the third place, we followed Kurdyumov et al. [14] using a slow-transition law to take the solution from the initial mass flow rate $\tilde{m}$ to the actual mass flow rate $m$ in which we would like to study the flame stability

$m=\tilde{m}+\Delta m \tanh (t / \tau)$,

where the parameter $\tau$ controls the transition time from $\tilde{m}$ to $m$ and is arbitrarily chosen to analyze it impact in the final solution. Concretely, in Fig. 13 we plot the evolution with time of $x_{f}, S$ for $m=3$ and $m=5$ and different values of $\tau$.

After analyzing the results, we can conclude that for all the mass flow rates tested, the flame behavior at $t \rightarrow \infty$ obtained for different initial conditions are the same and the only difference is the time at which the transition from symmetric $S=0$ to asymmetric $S \neq 0$ flames occurs, as can be seen by comparing Figs. 10, 12 and 13 . The above-described phenomena seems to be independent of the initial condition, at least for $\phi=0.5$, but the underlying

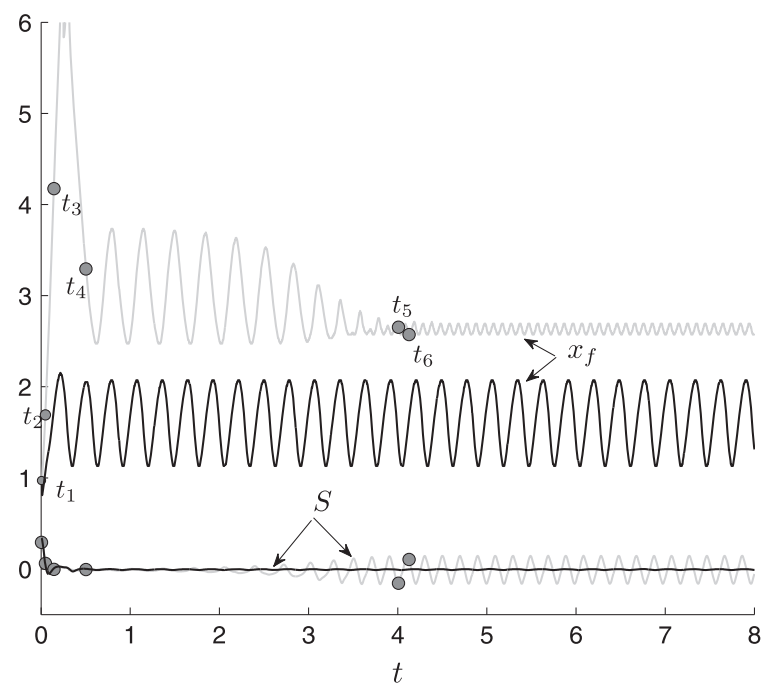

Fig. 12. The flame location $x_{f}$ and the symmetry parameter $S$ for $m=3$ (black) and $m=5$ (gray) vs. time obtained with the hot-spot initial condition given in Eq. (18) for $\phi=0.5, L e_{F}=1$. The symbols $\bullet$ indicate the time instants $t_{1} \cdots t_{6}$ where the isocontours are plotted in Fig. 5 in the case $m=5$.

physical reasons that explain this transition and the factors that determine the time at which it occurs are still to be understood.

\subsection{Case $L e_{F}=1.4$}

For a moderately large Lewis number $L e_{F}=1.4$, representative of fuels such as propane, the $m$ vs. $\phi$ stability map plotted in Fig. 14 reveals a flame that remains stable for a much wider range of mass flow rates in lean or slightly rich flames than that obtained for $L e_{F}=1$ or $L e_{F}=0.3$. A similar result was obtained by Norton and Vlachos [22] in their 2D computations of premixed propane flames. In his work, they claimed that this enhanced stability is caused by the lower propane's ignition temperature, which causes the reaction front to stabilize further upstream than for methane. Nevertheless, and since all the parameters but the Lewis number remain unmodified in our calculations, the lower propane's diffusivity must be the only responsible of the increasing flame stability for lean and stoichiometric mixtures.

As happened in the equidiffusional case, the stationary solution becomes unstable for every value of the equivalence ratio $\phi$ in the range of mass flow rates $m \in[0.351 .55]$, showing an oscillatory, 

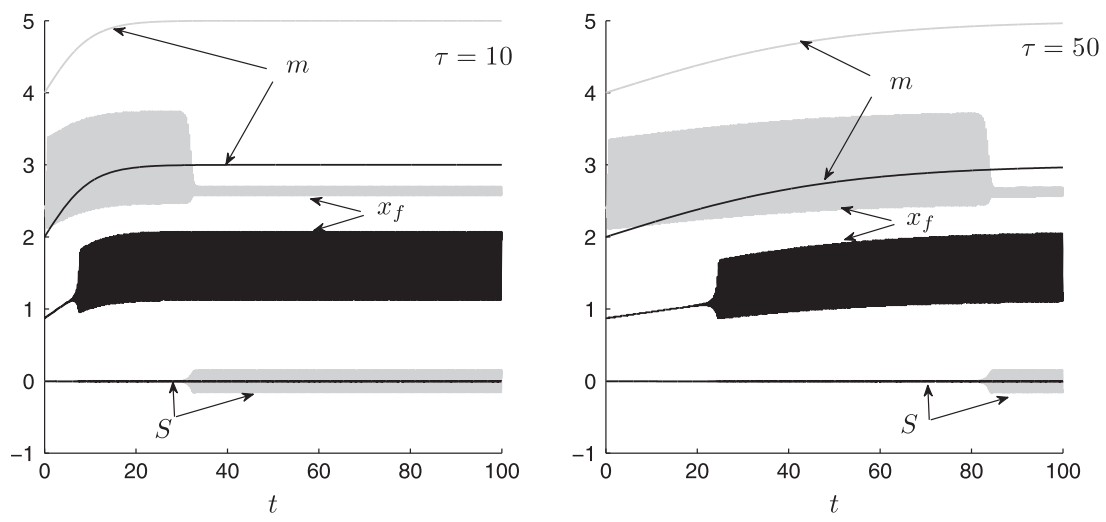

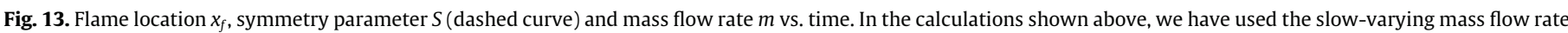
condition given in (21) with $\Delta m=1, m^{\sim}=2$ (black curves), $\sim m=4$ (gray curves), $\tau=10$ (left plot) and $\tau=50$ (right plot).

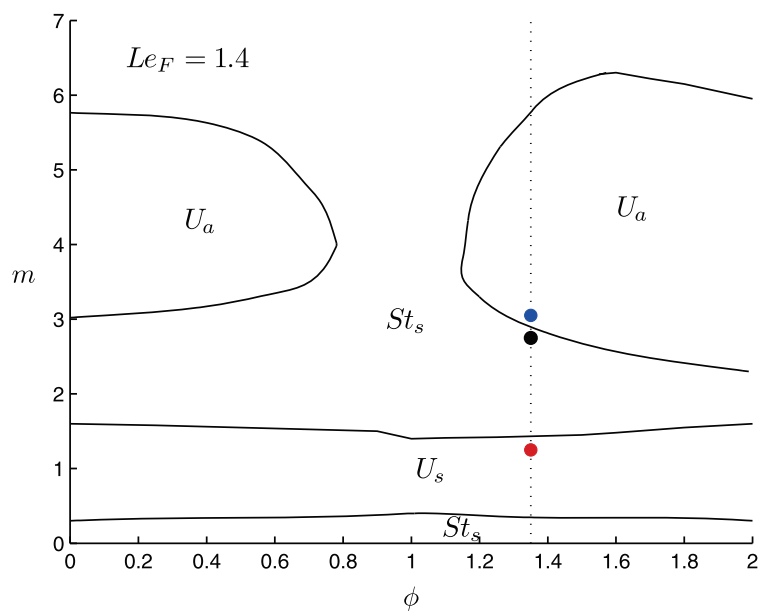

(a)

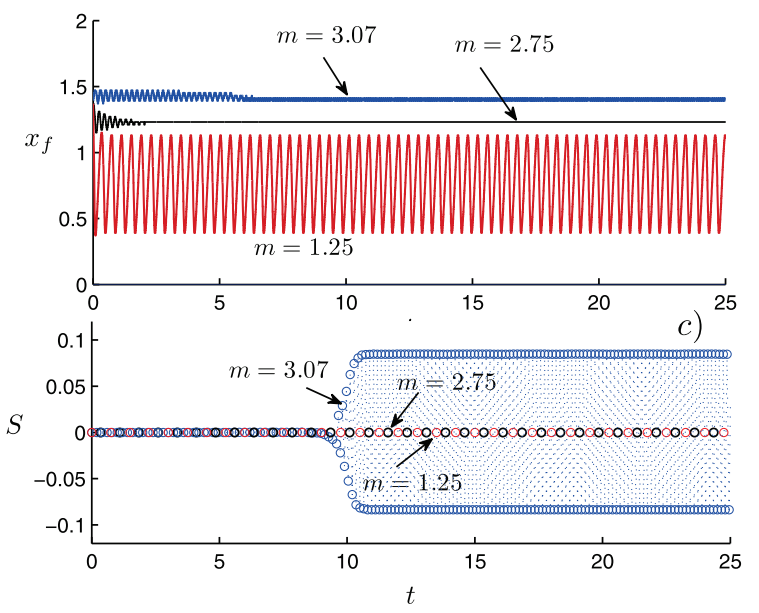

(b)

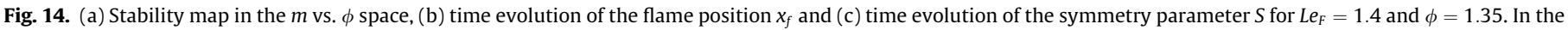

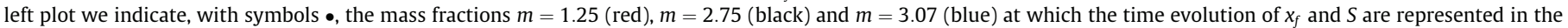
right plots. (For interpretation of the references to color in this figure legend, the reader is referred to the web version of this article.)

symmetric displacement than has been represented in Fig. 14 for $m=1.25$. The stability behavior in rich mixtures is seen to be similar to that of $L e_{F}=1$ with only quantitative difference in the threshold values of the mass flow rate separating stable from unstable flames.

As reported above for $L e_{F}=1$, there is a region of the stability map where the stationary solution losses its stability and initiate a symmetric, periodic oscillation that, after a relatively long period of time, becomes asymmetric reducing significantly the amplitude and increasing the frequency of the oscillation. An example of this behavior has been included in Fig. 14 for $m=3.07$ and $\phi=1.35$.

The analysis of the eigenvalues obtained with the linear stability analysis, shown in Fig. 15, determines very accurately the regions of stability and instability delimited by the values of the mass flow rate $m$ at which $\lambda_{r}<0$ or $\lambda_{r}>0$, respectively. Nevertheless, and similarly to the case $L e_{F}=1$, the prevalence of the symmetric or asymmetric modes at $t \rightarrow \infty$ can only be determined by the unsteady computations, as shown in Fig. 14 for $m=1.25, m=2.75$ and $m=3.07$ and $\phi=1.35$.

\section{The size matters: the effect of the Damkhôler number $d$}

The channel height is known to affect the stability of the flame. According to Pizza et al. [13] and Kurdyumov et al. [14], the flame

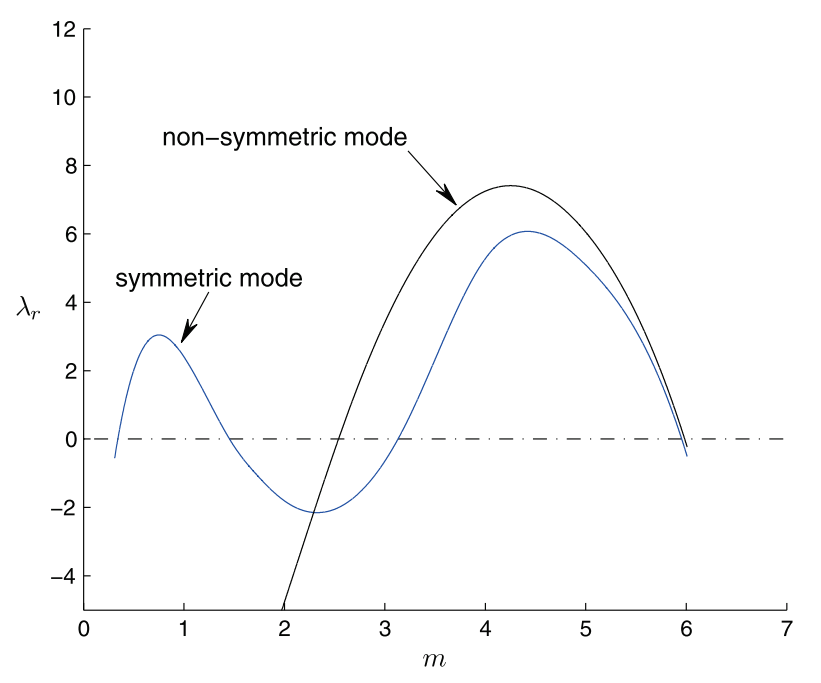

Fig. 15. Real eigenvalue $\lambda_{r}$ for the symmetric and non-symmetric modes vs. $m$ for $\phi=1.35$. In this case, the imaginary part of the eigenvalues is always positive $\lambda_{i}>0$, explaining why the curves are solid for the whole range of mass flow rates considered. 


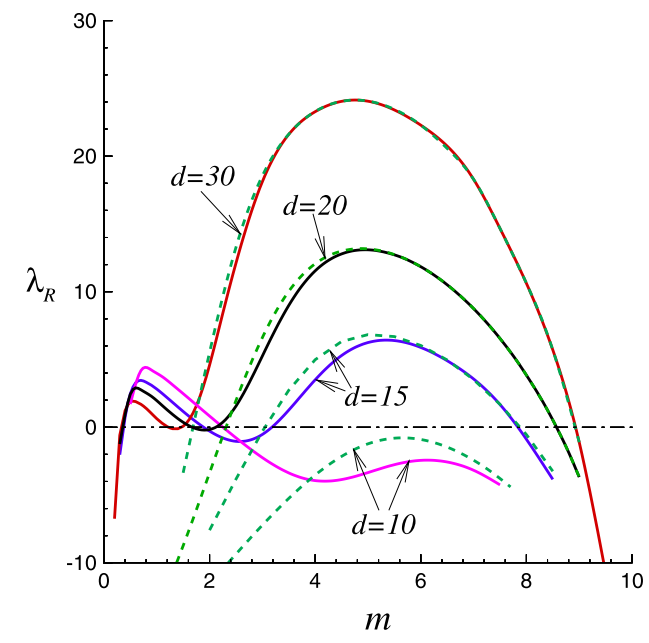

(a)

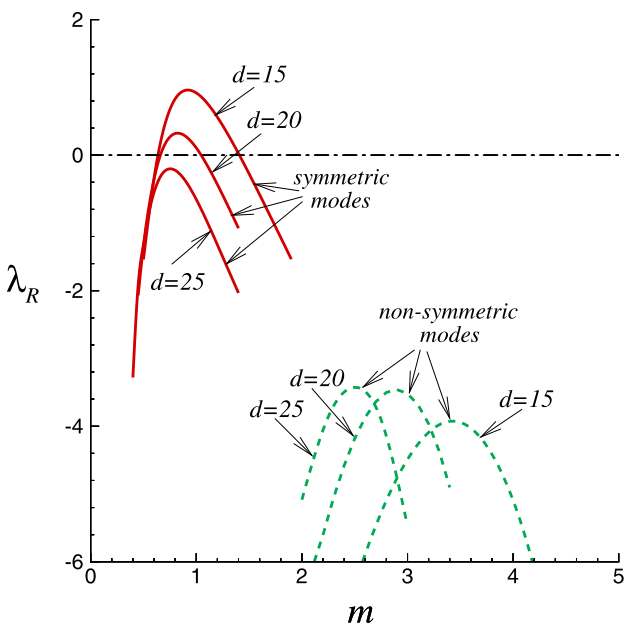

(b)

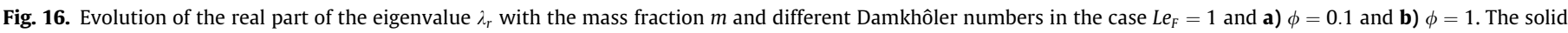
and dashed curves indicate symmetric and asymmetric modes, respectively.

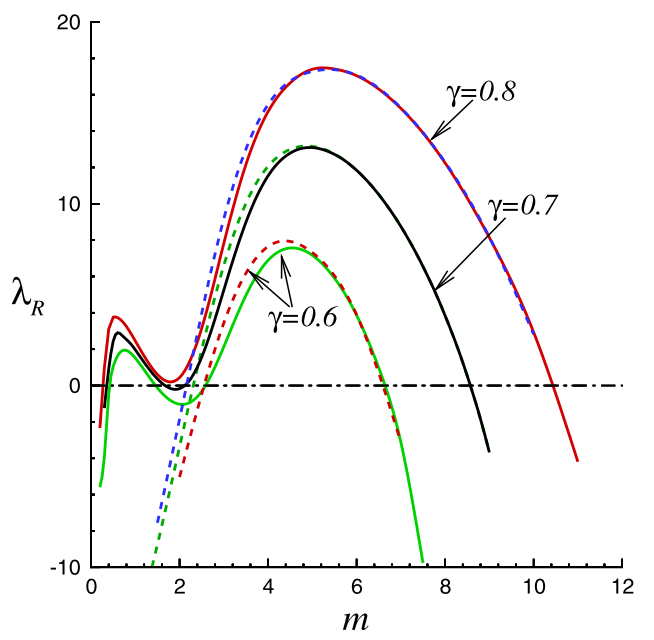

Fig. 17. Growth rate of the perturbation $\lambda_{r}$ vs. $m$ calculated with $d=20$, $\beta=10, \phi=0.1$ and three values of $\gamma$ for symmetric (solid lines) and non-symmetric (dashed lines) perturbation modes.

becomes more unstable in a wider range of mass flow rates as the height of the channel is increased in lean $\phi=0.5$ and very lean flames $\phi \rightarrow 0$, respectively. The same results have been obtained here by means of the stability analysis explained above. As can be seen in Fig. 16 for $L e_{F}=1$ and $\phi=0.1$, the range of mass flow rates at which the real part of the eigenvalue $\lambda_{r}$ is positive becomes wider as the channel width is increased, with larger maximum val-ues of $\lambda_{r}$ as $d$ increases. The eigenvalue $\lambda_{r}$ is almost identical for both asymmetric and symmetric modes and, therefore, from Fig. 16 we cannot anticipate the symmetric or asymmetric charac-ter of the flame oscillation that results from the development of the instability. As indicated in previous sections, only the unsteady calculation would determine if $S=0$ or $S \neq 0$ as $t \rightarrow \infty$.

The same calculation has been repeated in the case $L e_{F}=1$ and $\phi=1$ to get a completely different result. Unexpectedly, the flame becomes more stable for larger Damkhôler numbers $d$, being stable for all mass flow rates $m$ if $d$ is larger than approximately $d \simeq 22$ for both the symmetric and asymmetric modes, as can be checked in Fig. 16b). The eigenvalue $\lambda_{r}$ is, in this case, much smaller in the asymmetric than in the symmetric mode, explaining why perfectly symmetrical flame oscillations where obtained in previous section (see Fig. 10).

\section{The effect of the heat release $\gamma$}

Keeping constant the rest of the parameters of the problem, a change on the heat release parameter $\gamma$ can be understood as a variation of the dilution of the incoming fresh mixture. Even though the definition of $\gamma=\left(T_{s}-T_{0}\right) / T_{s}$ anticipates relatively small variations of this parameter, its effect on the stability of the flame cannot be neglected. For instance, for hydrogen, methane and propane flames, the parameter $\gamma$ oscillates between $\gamma=0.7$ for a stoichiometric ratio near the flammability limit $\phi \rightarrow 0$ and $\gamma=0.87$ near stoichiometric conditions $\phi=1$. In our calculations above, we chose $\gamma=0.7$ to reproduce the results obtained by previous works, most of them conceived within the frame of the lean mixtures approximation $\phi \rightarrow 0$, when the consumption of oxygen can be neglected and the conditions are close to the lean flammability limit. To measure the influence of this parameter in the stability of the flame, we have computed the variation of the eigenvalue $\lambda_{r}$ with $m$ for different values of the heat release parameter in the case $\phi=0.1$ to build Fig. 17. From that figure it is clear that the curves remain qualitatively similar, even though moderate quantitative changes were observed showing an increase of the unstable regions with increasing values of $\gamma$. Presumably this tendency is caused by the increase of the effective Zeldôvich number and the enhancement of the heat losses through the channel walls.

\section{Conclusions}

The effect of the equivalence ratio on the dynamics of a premixed flame propagating in a narrow channel with a step-wise wall temperature profile was investigated. A three-parameter study has been performed, where we have varied the incoming mass flow rate, the equivalence ratio and the fuel Lewis number in order to analyze their impact on the stability of the flame.

To delineate the stability map in a $m$ vs. $\phi$ parameter space, we have used two different strategies. Firstly, we have used a unsteady numerical computation initiated using two different initial conditions to assess its influence on the posterior evolution of the flame: a previously calculated steady solution and an artificial, hot-spot ignition. In second place, we carried out a classical eigenvalue 
analysis in which the steady symmetric base flow is slightly altered with a small-amplitude, harmonic perturbation.

These two strategies gave, as a result, the figures where we depicted, in a $m$ vs. $\phi$ parametric space, the flame dynamics identified for different fuel concentrations in the incoming, fresh mixture. According to these plots, very diffusive fuels $L e_{F}=0.3$ are unstable for both lean $\phi<1$ and rich flames $\phi>1$ in a large interval of incoming mass flow rates. The flame develops a periodical oscillation in which the symmetry of the flame depends on the initial condition of the computation. Near-stoichiometric mixtures, nevertheless, are seen to be stable for every $m$.

In the equidiffusional case $L e_{F}=L e_{0}=1$, the equations are symmetrical with respect to the equivalence ratio $\phi=1$ and, therefore, the stability behavior of rich and lean flames is coincident. Stoichiometric mixtures are more stable than richer or leaner mixtures, but it remains a range of mass flow rates in which an oscillatory instability develops.

For less diffusive fuels $L e_{F}=1.4$, the range of stability is much wider for lean and near-stoichiometric flames than for more diffusive fuels. For $\phi>1$, the stability behavior is similar to that described above for $L e_{F}=1$.

The last two sections of this work evidenced the importance of the channel size $d$ and the heat release parameter $\gamma$ in the stability behavior of the flame. While keeping the rest of parameters constant, an increase in $d$ for lean flames makes the flame considerably more unstable, confirming the findings of previous works. Nevertheless, as the stoichiometric ratio approaches $\phi=1$, that trend is completely reversed to give flames that become much more stable as the size of the channel is reduced. In the specific case shown in Fig. 16 where $\phi=1, L e_{F}=L e_{O}=1$, the flame becomes stable for every mass flow rate $m$ for a channel size below $d \simeq 22.5$.

On the other hand, as the heat release parameter $\gamma$ increases, the qualitative behavior of the flame remains equal, only with quantitative changes that show an increase of the unstable regions, presumably due to a larger effective Zeldôvich number and an increase of the heat losses through the channel walls.

\section{Acknowledgments}

This collaborative research was supported by the Spanish MCINN under Projects \#ENE2012-33213, \#ENE2011-27686-C0201 and CSD2010-00011 and by the local government of Comunidad de Madrid through Project \#S2009/ENE-1597.

\section{References}

[1] D.C. Walther, J. Ahn, Prog. Energy Combust. Sci. 37 (2011) 583-610.

[2] D. Dunn-Rankin, E. Martins Leal, David C. Walther, Prog. Energy Combust. Sci. 31 (2005) 422-465

[3] S.B. Sprague, S. Park, D.C. Walther, A.P. Pisano, C. Fernandez-Pello, Int. J. Altern. Propul. 1 (2007) 275-293.

[4] K. Fu, A. Knobloch, F. Martinez, D.C. Walther, C. Fernandez-Pello, A.P. Pisano, et al., In: IMECE/MEMS-23924, Proc. ASME 2001 International Mechanical Engineering Congress and Exposition (IMECE), New York, November 1116 2001.

[5] K. Fu, A. Knobloch, B. Cooley, D.C. Walther, D. Liepmann, C. Fernandez-Pello, K. Miyasaka. In: Proceedings of the 35th ASME 2001 National Heat Transfer Conference, Anaheim, CA, 2001. paper NHTC2001-11212.

[6] Y. Tsuji, B. Sprague, D. Walther, A. Pisano, C. Fernndez-Pello, AIAA Aerospace Sci. Meeting Exhibit (2005).

[7] Y. Ju, B. Xu, Proc. Combust. Inst. 30 (2005) 2445-2453.

[8] Y. Ju, K. Maruta, Prog. Energy Combust. Sci. 37 (2011) 669-715.

[9] K. Maruta, T. Kataoka, N.I. Kim, S. Minaev, R. Fursenko, Proc. Combust. Inst. 30 (2005) 2429-2936.

[10] H. Nakamura, A. Fan, H. Minamizono, K. Maruta, H. Kobayashi, T. Niioka, Proc. Combust. Inst. 32 (2009) 1367-1374

[11] F. Richecoeur, D.C. Kyritsis, Proc. Combust. Inst. 30 (2005) 2419-2427.

[12] C.J. Evans, D.C. Kyritsis, Proc. Combust. Inst. 32 (2009) 3107-3114.

[13] G. Pizza, C. Frouzakis, J. Mantzaras, A.G. Tomboulides, K. Boulouchos, Combust. Flame 152 (2008) 433-455.

[14] V.N. Kurdyumov, G. Pizza, C.E. Frouzakis, J. Mantzaras. Combust. Flame 156 (2009) 2190-2200.

[15] S.A. Lloyd, F.J. Weinberg, Nature 251 (1974) 47-49.

[16] A.R. Jones, S.A. Lloyd, F.J. Weinberg, Proc. R. Soc. London A 360 (1978) 97-

115.[17] P.D. Ronney, Combust. Flame 135 (2003) 421-439.

[18] Y. Ju, C.W. Choi, Combust. Flame 133 (2003) 483-493.

[19] M. Sánchez-Sanz, Combust. Flame 159 (2012) 3158-3167.

[20] J.L. Bechtold, M. Matalon, Combust. Flame 119 (1999) 217-232.

[21] V.N. Kurdyumov, Combust. Flame 158 (2011) 1307-1317.

[22] D.G. Norton, D.G. Vlachos, Combust. Flame 138 (2004) 97108. 